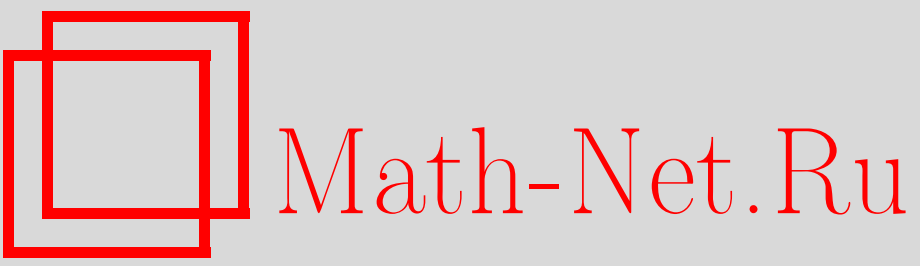

Р. С. Исмагилов, Слабые антикоммутационные соотношения и амальгамы грассмановых алгебр, ТМФ, 2000, том 125, номер 3, 425-431

DOI: https://doi.org/10.4213/tmf676

Использование Общероссийского математического портала Math-Net.Ru подразумевает, что вы прочитали и согласны с пользовательским соглашением

http://www.mathnet.ru/rus/agreement

Параметры загрузки:

IP: 54.162 .85 .209

26 апреля 2023 г., 17:33:58 


\section{СЛАБЫЕ АНТИКОММУТАЦИОННЫЕ СООТНОШЕНИЯ И АМАЛЬГАМЫ ГРАССМАНОВЫХ АЛГЕБР}

Рассматривается клиффордова алгебра, построенная по данному линейному пространству с симметрической билинейной формой, и в ней - семейство грассмановых подалгебр, порожденных всевозможными изотропными подпространствами. Описывается амальгама (индуктивный предел) этого семейства. В качестве приложения изучается модификация канонических антикоммутационных соотношений (KAC). Модификация состоит в том, что обычные $\mathrm{KAC}$ накладываются лишь на те пары векторов данного пространства, которые ортогональны относительно упомянутой формы (и каждый из векторов изотропен).

Канонические соотношения квантовой механики издавна служили поводом к математическим изысканиям. Предлагаемая работа содержит еше один пример такого рода. Краткое описание результатов содержится в [1].

\section{1. СЛАБЫЕ КАНОНИЧЕСКИЕ АНТИКОММУТАЦИОННЫЕ СООТНОШЕНИЯ. ПОСТАНОВКА ЗАДАЧИ}

Напомним известное определение канонических антикоммутационных соотношений (KAC), придав ему удобную для дальнейшего форму. Пусть $W$ - комплексное линейное пространство с невырожденной симметрической формой $\theta$. Невырожденность означает, что для любого вектора $x \in W, x \neq 0$, сушествует такой вектор $y \in W$, что $\theta(x, y) \neq 0$. Предположим, что в пространстве $W$ задана инволюция $x \mapsto \bar{x}$, причем $\theta(x, \bar{x})>0$, если $x \neq 0$. Пусть $H$ - гильбертово пространство.Тогда КАС задаются семейством ограниченных линейных операторов $C(x), x \in W$, действующих в $H$ и удовлетворяющих условиям

$$
\begin{aligned}
& C(\bar{x})=(C(x))^{*}, \quad C(\alpha x+\beta y)=\alpha C(x)+\beta C(y), \quad \alpha, \beta \in \mathbf{C}, \\
& \{C(x), C(y)\}=\lambda \theta(x, y) I
\end{aligned}
$$

для некоторого числа $\lambda \geqslant 0$. Как обычно, $\{A, B\}$ означает антикоммутатор, а параметр $\lambda$ введен для удобства.

\footnotetext{
* Московское высшее техническое училище им. Н.Э. Баумана, Москва, Россия
} 
Модифищируем это определение. Модификация будет состоять в том, что мы потребуем выполнения соотношений $(1),(2)$ лишш для векторов $x, y \in W$, которые ортогональны относительно формы $\theta$ и, кроме того, каждый из них ортогонален самому себе. Это приведет нас к понятию cлабыx KAC. Точное определение таково. Возьмем те же $W, \theta, H$, что и выше. В дальнейшем ортогональность векторов $x, y \in W$ относительно формы $\theta$ (т.е. равенство $\theta(x, y)=0$ ) будем записывать в виде $x \perp y$. Введем множество $W^{\circ}=\{x: x \in W, x \perp x\}$. Пусть семейство операторов $\left\{C^{\circ}(x), x \in W^{\circ}\right\}$ удовлетворяет следующему условию: если

$$
x, y \in W, \quad x \perp x, \quad y \perp y, \quad x \perp y,
$$

то

$$
\begin{gathered}
C^{\circ}(\alpha x+\beta y)=\alpha C^{\circ}(x)+\beta C^{\circ}(y), \quad\left\{C^{\circ}(x), C^{\circ}(y)\right\}=0, \quad \alpha, \beta \in \mathbf{C}, \\
C^{\circ}(\bar{x})=\left(C^{\circ}(x)\right)^{*} .
\end{gathered}
$$

В этом случае будем говорить,что семейство $\left\{C^{\circ}(x)\right\}, x \in W^{\circ}$, удовлетворяет $c л a-$ бым. $\mathrm{KAC}$.

Ясно, что из обычных КАC вытекают слабые КАС. Мы увидим, что верно и обратное, если только рассматриваемое семейство операторов $\left\{C^{\circ}(x)\right\}$ неприводимо. Точная формулировка результата содержится в следуюшей теореме.

ТЕОРема 1. Пусть семейство $C^{\circ}(x), \quad x \in W^{\circ}$, удовлетворяет слабим КАС и неприводимо. Тогда существует (и единственно) семейство ограниченных операторов $C(x), x \in W$, которое удовлетворяет $K A C$ (т.е.соотношениям (1), (2) для некоторого $\lambda \geqslant 0)$ и условиям $C(x)=C^{\circ}(x), \quad x \in W^{\circ}$.

Чтобы пояснить сущность этого результата, рассмотрим более общую ситуацию. Пусть $K$ - алгебраически замкнутое поле с характеристикой, отличной от 2, и $W$ линейное пространство над $K$, снабженное симметричной невырожденной билинейной формой $\theta$. Введем множество $W^{\circ}=\{x: x \in W, x \perp x\}$. Пусть $B$ - ассоциативная алгебранад $K$, обладающая единищей. Рассмотрим произвольное отображение $f^{\circ}: W^{\circ} \rightarrow B$, удовлетворяющее условию, которое аналогично слабым КАС (с тем, впрочем, отличием, что теперь в пространстве $W$ нет инволюции), т.е., если

$$
x, y \in W, \quad x \perp x, \quad y \perp y, \quad x \perp y,
$$

то

$$
f^{\circ}(\alpha x+\beta y)=\alpha f^{\circ}(x)+\beta f^{\circ}(y), \quad\left\{f^{\circ}(x), f^{\circ}(y)\right\}=0, \quad \alpha, \beta \in K .
$$


ТЕОРЕма 2. Существуют (и однозначно определень) линейное отобрахсение $f: W \rightarrow A$ и әлемент $b \in B$, удовлетворяющие условиям

$$
\begin{aligned}
\{f(x), f(y)\} & =\theta(x, y) b, & & x, y \in W, \\
f^{\circ}(x) & =f(x), & & x \in W^{\circ} .
\end{aligned}
$$

Элемент $b$ перестановочен со всеми $f(x), x \in W$.

Ясно, что теорема 1 легко выводится из теоремы 2 (то обстоятельство, что в теореме 2 отсутствует инволюция, не препятствует такому выводу).

Прежде чем переходить к доказательству, приведем еше одну формулировку теоремы 2 , пользуясь понятием амальгамы семейства алгебр. Это будет сделано в разделе 2. Описание этой амальгамы и есть основной результат работы; применение к КАС - лиш простая иллюстрация этого результата.

\section{2. АМАЛЬГАМЫ СЕМЕЙСТВА АЛГЕБР. СЛУЧАЙ СЕМЕЙСТВА ГРАССМАНОВЫХ ПОДАЛГЕБР АЛГЕБРЫ КЛИФФОРДА}

Начнем с определений. Пусть $\left\{A_{\alpha}\right\}$ - набор ассоциативных алгебр с единицей (всюду в этом разделе рассматриваются только такие алгебры). Предположим, что для некоторых пар $\alpha, \beta$ указан гомоморфизм $\phi_{\alpha \beta}: A_{\alpha} \rightarrow A_{\beta}$, что запишем в виде $\left\{A_{\alpha}, \phi_{\alpha \beta}\right\}$. Назовем представлением этого семейства $\left\{A_{\alpha}, \phi_{\alpha \beta}\right\}$ в некоторой алгебре $B$ такой набор гомоморфизмов $f_{\alpha}: A_{\alpha} \rightarrow B$ (по одному гомоморфизму для каждой алгебры $A_{\alpha}$ ), что $f_{\alpha}=f_{\beta} \circ \phi_{\alpha \beta}$ для всех $\alpha, \beta$, для которых определен $\phi_{\alpha \beta}$, и, кроме того, множество $\cup f_{\alpha}\left(A_{\alpha}\right)$ порождает алгебру $B$. Данное представление записываем кратко в виде $B,\left\{f_{\alpha}\right\}$. Амальгама семейства $\left\{A_{\alpha}, \phi_{\alpha \beta}\right\}$ определяется как представление $B^{1},\left\{f_{\alpha}^{1}\right\}$, обладающее следующим свойством универсальности: для любого представления $B,\left\{f_{\alpha}\right\}$ сушествует такой гомоморфизм алгебр $p: B^{1} \rightarrow B$, что $f_{\alpha}=p \circ f_{\alpha}^{1}$ для всех $\alpha$ (употребляются также термины "амальгамированное произведение" и “индуктивный предел"). Иногда (для краткости) амальгамой будем называть алгебру $B^{1}$.

Отметим частный случай, наиболее важный для дальнейшего. Пусть даны алгебра $A$ и набор ее подалгебр $A_{\alpha}$, замкнутый относительно пересечений и порождающий алгебру $A$. Если $A_{\alpha} \subset A_{\beta}$, то определен гомоморфизм включения $A_{\alpha} \rightarrow A_{\beta}$. Итак, мы получили семейство, состоящее из алгебр и гомоморфизмов включения; для краткости будем записывать его в виде $\left\{A_{\alpha}\right\}$ (опуская упоминание о гомоморфизмах). Теперь можно поставить задачу вычисления амальгамы семейства $\left\{A_{\alpha}\right\}$.

Приведенное определение заимствовано из теории групп, где оно служит важным средством исследования; обстоятельное изложение этого вопроса содержится в работе [2].

Сейчас мы обратимся к примеру амальгамы, связанному с задачей, описанной в разделе 1. Итак, вернемся к линейному пространству $W$ (над полем $K$, характеристика которого отлична от 2), снабженному невырожденной симметрической билинейной формой $\theta$. Линейное подпространство $X \subset W$ называется изотропнылм (иногда вполне 
изотропныц $)$, если $\theta(x, y)=0$ для всех $x, y \in X$; множество всех изотропных подпространств обозначим через $I s(W)$.

Напомним, что алгебра Клиффорда $C(W)$ определяется линейным пространством $W$ и соотношением $x y+y x=\theta(x, y) e$, где $e$ - единица алгебры. Любое изотропное подпространство $X \subset W$ порождает в $C(W)$ грассманову подалгебру $\operatorname{Gr}(X)$; последняя задается пространством $X$ и соотношением $x y+y x=0$. Итак, мы пришли к семейству грассмановых алгебр $\{G r(X)\}, X \in I s(W)$ (гомоморфизм-включение $\operatorname{Gr}(X) \rightarrow G r(Y)$ определен при $X \subset Y)$. Задача состоит в нахождении его амальгамы.

Чтобы изложить решение этой задачи, рассмотрим алгебру всех многочленов

$$
\alpha_{0}+\sum_{k \geqslant 1} c_{k} T^{k}, \quad \alpha_{0} \in K, \quad c_{k} \in C(W),
$$

с обычными алгебраическими операциями (сумма в (3) конечна). Таким образом, формальная переменная $T$ перестановочна с элементами алгебры $C(W)$. Обозначим через $C(W, T)$ подалгебру, порожденную одночленами $c_{1} T, c_{1} \in W$. Легко описать явно эту подалгебру. Для этого напомним, что алгебра $C(W)$ допускает фильтрацию $C_{0}(W) \subset C_{1}(W) \subset \cdots$, где $C_{k}(W), k>0$, есть линейная оболочка элементов алгебры $C(W)$, представимых в виде произведения не более чем $k$ элементов из $W$. Имеем также разложение в прямую сумму линейных пространств $C(W)=C^{+}(W) \oplus C^{-}(W)$, где $C^{+}(W)$ и $C^{-}(W)$ - линейные оболочки упомянутых произведений с четным и соответственно нечетным числом сомножителей. Легко видеть, что $C(W, T)$ состоит из всех многочленов вида (3), где $c_{k} \in C_{k}(W) \cap C^{+}(W)$ при четном $k, c_{k} \in C_{k}(W) \cap C^{-}(W)$ при нечетном $k$.

Для любого подпространства $X \in I s(W)$ вложение линейных пространств $X \rightarrow C^{\circ}(W, T), \quad x \mapsto x T$, продолжается до гомоморфизма алгебр $f_{X}^{\circ}: G r(X) \rightarrow$ $C(W, T)$. Тем самым получилось представление семейства алгебр $\{G r(X)\}, X \in I s(W)$, в алгебре $C(W, T)$. Выше мы условились записывать его в виде $C(W, T),\left\{f_{X}^{\circ}\right\}$.

Теорема 3. Алгебра $C(W, T)$ (с гомоморфизмами $f_{X}^{\circ}: G r(X) \rightarrow C(W, T)$ ) есть амальгама семейства $\{\operatorname{Gr}(X), X \in I s(W)\}$.

Следуюшее простое предложение дает еше одно описание алгебры $C(W, T)$.

ПредлоЖенИЕ 1. Алгебра $C(W, T)$ изоморфна алгебре, которая порождена линейнымм пространством $W$ и формальной переменной $S$, связанными соотношениями

$$
x y+y x=\theta(x, y) S, \quad x S=S x, \quad x, y \in W ;
$$

изоморфизм устанавливается по правилам $x \mapsto x T, S \mapsto T^{2}$.

Это предложение равносильно следующему. Пусть $D$-произвольная $K$-алгебра (как всегда, ассоциативная и имеюшая единицу). Пусть даны линейное отображение $\phi: W \rightarrow D$ и элемент $d \in D$ такие, что

$$
\phi(x) \phi(y)+\phi(y) \phi(x)=\theta(x, y) d, \quad \phi(x) d=d \phi(x), \quad x, y \in W .
$$

Тогда сушествует такой гомоморфизм алгебр $p: C(W, T) \rightarrow D$, что $\phi(x)=p(x T), d=$ $p\left(T^{2}\right)$. 


\section{3. ДОКАЗАТЕЛЬСТВА}

Начнем с доказательства предложения 1 (точнее, только что сформулированного эквивалентного утверждения). Пусть сначала $\operatorname{dim} W=n<\infty$. Тогда, как известно, в $W$ существует такой базис $\left\{e_{k}, 1 \leqslant k \leqslant n\right\}$, что $\theta\left(e_{i}, e_{k}\right)=\delta_{i k}, 1 \leqslant i, k \leqslant n$. Для любого $k \in\{1, \ldots, n\}$ элементы $e_{i_{1}} \cdots e_{i_{k}}$ образуют базис линейного пространства $C_{k}(W)$. Поэтому существует такое линейное отображение $p: C(W, T) \rightarrow D$, что $p\left(e_{i_{1}} \ldots e_{i_{k}} T^{r}\right)=$ $\phi\left(e_{i_{1}}\right) \ldots \phi\left(e_{i_{k}}\right) d^{(r-k) / 2}$ для четных $r-k \geqslant 0$. Без труда проверяется, что построенное отображение есть искомый гомоморфизм. Кроме того, этими двумя свойствами отображение $p$ однозначно определено. Это позволяет построить нужный гомоморфизм $p$ также для случая $\operatorname{dim} W=\infty$. Этим доказано предложение 1 .

Переходим к доказательству теоремы 2.

Лемма 1. Пусть $a, b, c \in W^{\circ}, \theta(b, c) \neq 0$. Тогда существуют такие $x, y \in W^{\circ}$, что $a=x+y, x \perp b, y \perp c$ (из этих условий следует, что $x \perp y)$.

ДокАЗАТЕЛЬСТВО. Достаточно взять векторы

$$
x=\frac{a}{2}+s b+t c+d, \quad y=\frac{a}{2}-s b-t c-d,
$$

где

$$
s=\theta(a, c)(2 \theta(b, c))^{-1}, \quad t=-\theta(a, b)(2 \theta(b, c))^{-1},
$$

а вектор $d$ удовлетворяет условиям

$$
d \perp a, \quad d \perp b, \quad d \perp c, \quad \theta(d, d)=-2 s t \theta(b, c) .
$$

Сушествование вектора $d$ с этими свойствами вытекает из условия $\operatorname{dim} W \geqslant 4$ и алгебраической замкнутости поля $K$. Лемма доказана.

Лемма 2. Отображение $f^{\circ}: W^{\circ} \rightarrow B$ продолжсается до линейного отображения $f: W \rightarrow B$.

ДокАЗАТЕЛЬСтво. Достаточно проверить, что если $a_{i} \in W^{\circ}, 1 \leqslant i \leqslant p, a_{1}+\cdots$ $\cdots+a_{p}=0$, то

$$
f^{\circ}\left(a_{1}\right)+\cdots+f^{\circ}\left(a_{p}\right)=0 .
$$

При $p=2$ это очевидно. Пусть $p>2$ и пусть данное утвержение доказано при $p^{\prime}<p$. Если все векторы $a_{i}$ попарно ортогональны (напомним также, что каждый из них ортогонален самому себе), то утверждение вытекает из определения отображения $f^{\circ}$. Пусть теперь среди векторов $a_{i}$ имеется неортогональная пара. Можно считать, что $\theta\left(a_{2}, a_{3}\right) \neq$ 0 . Согласно лемме $1, a_{1}=x+y$, где $x \perp x, x \perp y, y \perp y, x \perp a_{2}, y \perp a_{3}$. Тогда $\left(x+a_{2}\right)+\left(y+a_{3}\right)+\cdots+a_{p}=0$, причем векторы $x+a_{2}, y+a_{3}, \ldots, a_{p}$ принадлежат множеству $W^{\circ}$. По предположению индукции $f\left(x+a_{2}\right)+f\left(y+a_{3}\right)+\cdots+f\left(a_{p}\right)=0$. Так как $x \perp a_{2}, y \perp a_{3}, x \perp x, y \perp y, x \perp y$, то $f\left(x+a_{2}\right)+f\left(y+a_{3}\right)=f(x)+f\left(a_{2}\right)+f(y)+$ $f\left(a_{3}\right)=f(x+y)+f\left(a_{2}\right)+f\left(a_{3}\right)=f\left(a_{1}\right)+f\left(a_{2}\right)+f\left(a_{3}\right)$. Отсюда следует равенство (4). Лемма доказана. 
Лемма 3. Пусть $f: W \rightarrow B$ - линейное отображсение из леммы 2. Тогда существует такой элемент $b \in B$, что $b f(x)=f(x) b, \quad\{f(x), f(y)\}=\theta(x, y) b, x, y \in W$.

ДокАЗАТЕЛЬСтво. Покажем, что если $x, y \in W, \theta(x, x)=\theta(y, y)$, то $\{f(x), f(x)\}=$ $\{f(y), f(y)\}$. Пусть сначала $x \perp y$. Возьмем такой элемент $\epsilon \in K$, что $\epsilon^{2}=-1$. Тогда $(x+\epsilon y) \perp(x+\epsilon y)$, откуда следует, что

$$
\{f(x+\epsilon y), f(x+\epsilon y)\}=\{f(x), f(x)\}-\{f(y), f(y)\}+2 \epsilon\{f(x), f(y)\}=0 .
$$

Это соотношение остается верным при замене $\epsilon$ на $-\epsilon$. Сложив два соотношения, получаем равенство $\{f(x), f(x)\}=\{f(y), f(y)\}$, что и утверждалось. Если же условие $x \perp y$ нарушено, то возьмем вектор $z \in W, \theta(z, z)=\theta(x, x)=\theta(y, y)$, ортогональный к $x, y$, и применим это рассуждение к парам $(x, z),(y, z)$. Этим утверждение доказано.

Таким образом, сушествует такой элемент $b \in B$, что $\{f(x), f(x)\}=b$ при $\theta(x, x)=1$. Но тогда $\{f(x), f(x)\}=\theta(x, x) b, \quad x \in W$, откуда (применяя стандартный переход от квадратичного соотношения к билинейному) получаем равенство $\{f(x), f(y)\}=$ $\theta(x, y) b, x, y \in W$. Ясно, что элемент $b$ перестановочен с $f(x), \theta(x, x)=1$, а потому и с любым $f(x), x \in W$ (ибо $W$ есть линейная оболочка векторов $x, \theta(x, x)=1$ ). Лемма 3 доказана.

Теперь теорема 2 вытекает из предложения 1 (точнее, из равносильного ему утверждения, сформулированного после предложения 1) и леммы 3.

Наконец, теорема 3 следует из теоремы 2 (и определения амальгамы).

\section{4. СЛАБЫЕ КАНОНИЧЕСКИЕ КОММУТАЦИОННЫЕ СООТНОШЕНИЯ}

В этом разделе мы приведем ради полноты изложения результаты, касающиеся слабых канонических коммутационных соотношений (KKC).

Пусть $V$ - вешественное линейное пространство с невырожденной кососимметрической формой $\tau$ (невырожденность означает, что если $x \in V, x \neq 0$, то $\tau(x, y) \neq 0$ для некоторого $y \in V), H$ - гильбертово пространство с плотным линейным подмножеством $D$. Пусть, далее, каждому $x \in V$ поставлен в соответствие симметрический оператор $A(x)$ в $H$, отображающий множество $D$ в $D$ и удовлетворяющий следующему условию: если

$$
x, y \in V, \quad x \perp y,
$$

то

$$
A(\alpha x+\beta y)=\alpha A(x)+\beta A(y), \quad \alpha, \beta \in \mathbf{R}, \quad[A(x), A(y)]=0 .
$$

Как обычно, $[A, B]$ - это коммутатор $A B-B A$; запись $x \perp y$ означает $\tau(x, y)=0$.

Подчеркнем, что здесь мы не требуем линейности отображения $x \mapsto A(x)$; равенство, выражающее линейность, а также равенство, выражающее $\mathrm{KKC}$, должны выполняться лишь для любой ортогональной (относительно $\tau$ ) пары векторов из $V$. В этом случае 
мы скажем, что семейство удовлетворяет слабым ККС. Напомним, что обычные ККС выражаются формулами

$$
\begin{aligned}
& A(\alpha x+\beta y)=\alpha A(x)+\beta A(y), \quad x, y \in V, \quad \alpha, \beta \in \mathbf{R}, \\
& {[A(x), A(y)]=\lambda \tau(x, y) I, \quad x, y \in V}
\end{aligned}
$$

Заметим, что в эти соотношения включен вешественньй параметр $\lambda$; при $\lambda=0$ дело сводится к коммутативности семейства $\{A(x)\}$.

Разумеется, из обычных ККС вытекают слабые КKC. Верно ли обратное? Ответ на этот вопрос утвердителен, если выполняется следуюшее условие операторной неприводимости: любой линейный оператор в $D$, которьй перестановочен со всеми операторами $A(x)$ и выражается в виде многочлена не выше второй степени от некоторого конечного набора этих операторов, скалярен.

Аналогичный результат справедлив и для KKC в интегральной форме. Эти результаты получены в работах $[1,3]$ как следствие явного описания индуктивных пределов амальгам некоторых семейств абелевых групп и алгебр Ли. В частности, здесь возникает задача о вычислении амальгамы семейства всех изотропных относительно формы $\tau$ линейных подпространств $X \subset V$ (каждое такое подпространство понимается как абелева группа по отношению к сложению векторов). Оказьвается, что искомая амальгама - это группа Гейзенберга, построенная обычным образом по $V, \tau$. Другие примеры вычисления амальгам содержатся в работах $[4,5]$.

Благодарности. Работа поддержана Российским фондом фундаментальных исследований (грант № 96-15-96249).

\section{Список литературы}

[1] Р. С. Исмагилов. Функц. анализ и его прилож. 2000. Т. 34. Вып. 2. С. 75-78.

[2] Ж. П. Серр. Деревья, амальгамы и математика. Периодич. сб. переводов иностр. статей. 1974. T. 18. № 1. C. $3-51$.

[3] Р. С. Исмагилов. Вестн. Тамбовского ун-та. Сер. Естественные и технич. науки. 1997. Т. 2. № 4. С. $367-361$.

[4] Р. С. Исмагилов. Матем. сб. 2000. Т. 191. № 3. С. 53-64.

[5] Р. С. Исмагилов. Изв. АН СССР. Сер. матем. 1980. Т. 44. С. 831-867.

Поступила в редакцию $14 . \mathrm{IV} .2000$ г. 